\title{
ATOMISTIC SIMULATIONS OF THE MOTION OF AN EDGE DISLOCATION IN ALUMINUM USING THE EMBEDDED ATOM METHOD
}

\author{
N. Bhate, R. J. Clifton and R. Phillips* \\ Division of Engineering, Brown University, Providence, Rhode Island 02912 \\ ${ }^{*}$ Graduate Aeronautical Laboratories, Caltech, Pasadena, California 91125
}

\begin{abstract}
The motion of an edge dislocation is analyzed for temperatures ranging from $10 \mathrm{~K}$ to 200 $\mathrm{K}$ and for stresses up to $5 \mathrm{GPa}$. The dislocation velocity versus the applied shear stress curve can be divided into four regimes corresponding to successively higher shear stresses. In the first regime, the applied shear stress is below the Peierls stress and the dislocation velocity is nominally zero. In the second regime, the dislocation velocity decreases with increasing temperature indicating the presence of a drag due to thermal phonons. In the third regime, the dislocation reaches a sub-sonic limiting velocity that can be predicted by a two-dimensional lattice-dynamics analysis. Such an analysis predicts a limiting velocity for a moving defect when the phase velocity and the group velocity are equal. If even higher shear stresses are applied, the dislocation travels with a transonic velocity of $\sqrt{2}$ times the shear wave speed.
\end{abstract}

\section{INTRODUCTION}

Improved understanding of the mobility of dislocations is important to improved understanding of the mechanical behavior of crystalline materials. While for many structural alloys the motion of dislocations is limited primarily by the presence of interstitials, solute atoms, and precipitates, there are cases for which the intrinsic resistance of the lattice plays a dominant role in limiting the motion of dislocations. Such cases include the plastic behavior of $\mathrm{Ni}_{3} \mathrm{Al}$ with its $\mathrm{L}_{2}$ crystal structure $^{1}$ and the brittle versus ductile failure of tungsten single crystals at different temperatures ${ }^{2}$. Although most interest is in the subsonic motion of dislocations, there are phenomena - such as martensitic twinning and geophysical fault slip - where the transonic or supersonic motion of dislocations appears to be of interest. Several researchers have simulated the dynamics of dislocations, with particular emphasis on probing the high velocity regime $e^{3,4,5}$. In the present study, molecular dynamics is used to examine the effects of applied stress and temperature on the mobility of an edge dislocation in $\mathrm{Al}$.

\section{METHODOLOGY AND MODEL DESCRIPTION}

The simulation cell geometry for modeling an edge dislocation in $\mathrm{Al}$ is shown in Figure 1. The crystal is oriented so that the $\mathrm{X}$-axis is along the slip direction $\langle 110\rangle$, the Y-axis is along the dislocation line $\langle 112\rangle$ and the Zaxis is oriented along the normal $\langle 111\rangle$ to the slip plane. The cell dimensions are $226.66 \AA \times$ $4.59 \AA \times 137 \AA$ or $40 \times 2 \times 60$ atomic layers in the $\mathrm{X}, \mathrm{Y}$ and $\mathrm{Z}$ directions, respectively. Periodic boundary conditions are imposed at cell boundaries that are perpendicular to the $\mathrm{X}$ and the $\mathrm{Y}$ directions. The cell is regarded as being finite along the $\mathrm{Z}$ direction. Interaction forces between atoms are described by the embedded atom method $(\mathrm{EAM})^{4}$, using potentials for 


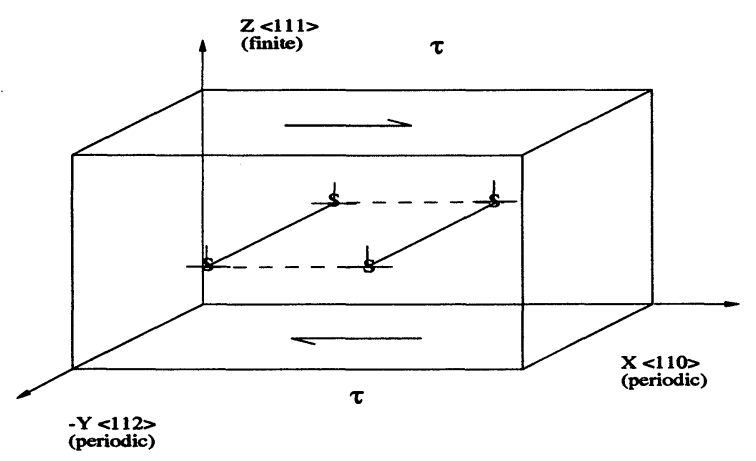

FIGURE 1: Schematic of the simulation cell.

aluminum developed by Ercolessi and Adams ${ }^{7}$.

The simulation cell is constructed as a perfect aluminum lattice. Two atomic half-layers are removed from the lower half of the cell. The remaining atoms are then displaced by the Volterra displacements in order to introduce an edge dislocation. The energy of the configuration is minimized subject to the requirement that the atoms in the top and bottom layers move only in the $\mathrm{X}-\mathrm{Y}$ plane. The energy minimization results in dissociation of the edge dislocation into two partials separated by a stacking fault. The width of the stacking fault agrees well with that obtained using lattice statics. The configuration with the dissociated edge dislocation is used as the initial configuration for all of the molecular dynamics simulations.

Simulations have been carried out at four temperatures: $10 \mathrm{~K}, 50 \mathrm{~K}, 100 \mathrm{~K}$ and $200 \mathrm{~K}$, and for shear stresses ranging from $1 \mathrm{MPa}$ to $5000 \mathrm{MPa}$. At each temperature, the atoms in the initial configuration are given random initial velocities corresponding to the MaxwellBoltzmann distribution at the test temperature. Temperature is maintained constant in the simulations by applying the Nose-Hoover drag ${ }^{8}$.

The initial configuration is allowed to equilibrate for $1 \mathrm{ps}$ in order to achieve equipartitioning of the energy. An equal and opposite shear stress is then applied to the top and bottom surfaces by applying forces to the atoms in the top and bottom layers. The simulation is carried out for a total of $100 \mathrm{ps}$ under application of the constant stress. The edge dislocation is observed to reach

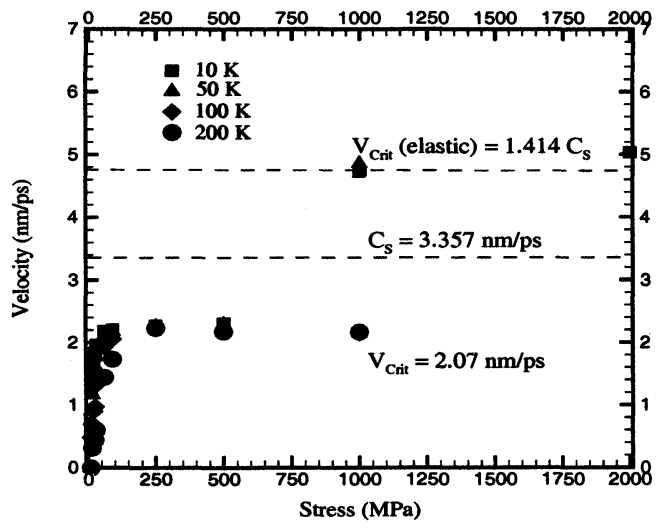

FIGURE 2: Dislocation velocity as a function of applied stress and temperature.

a steady velocity within the first $20-40 \mathrm{ps}$. The rise time corresponds to a few multiples of the time, $t_{h} \approx 4 p s$, taken by an elastic shear wave to travel the thickness of the film.

\section{COMPUTATIONAL RESULTS}

The position of the edge dislocation as a function of time is determined from the location of the peak in the slip distribution. It is clear from plotting dislocation position vs. time for various stresses and temperatures that the resistance to the motion of the dislocation is higher at higher temperatures, although at a shear stress of 500 $\mathrm{MPa}$, the effect of temperature appears to be negligible. At the highest temperature, $200 \mathrm{~K}$, and the lowest stress, $10 \mathrm{MPa}$, the dislocation is even arrested momentarily. In all cases, the dislocation approaches a steady velocity after $40 \mathrm{ps}$. The steady velocity reached for these edge dislocations is shown in Figures 2 and 3 as a function of the applied stress at each of the four temperatures. From the figures it is evident that there are four distinct regimes. In the first regime, the shear stress is sufficiently small that the dislocation is stationary, or essentially so. The behavior in this regime is governed by the Peierls barrier. In the second regime, the shear stress is large enough to cause dislocation motion - at a velocity that decreases with increasing temperature. In this regime the behavior is strongly affected by the 


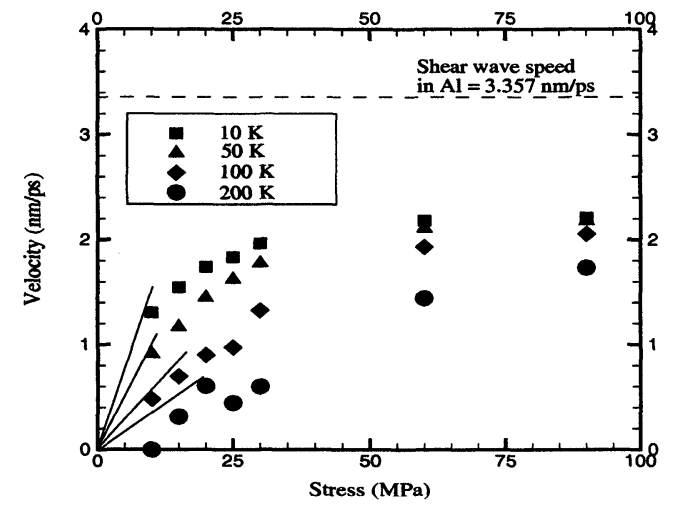

FIGURE 3: Tangent lines, drawn from the origin, to estimate the dislocation drag coefficient.

interaction of dislocations with thermal phonons. At still higher stresses, say greater than 100 $\mathrm{MPa}$, the curves in Figure 2 approach a plateau corresponding to a temperature-independent, limiting dislocation velocity of approximately $2.1 \mathrm{~nm} / \mathrm{ps}$, or approximately $70 \%$ of the speed of elastic shear waves in aluminum. An explanation of this limiting velocity is provided in the final section of this paper. Finally, at the highest shear stresses shown, there is a fourth regime in which the dislocation velocity jumps to a transonic speed of approximately $\sqrt{2} c_{s}$ where $c_{s}$ is the elastic shear wave speed. Eshelby ${ }^{9}$ has established that - in continuum elasticity transonic, radiation-free dislocation motion can occur only at a dislocation velocity of $\sqrt{2} c_{s}$.

\section{DISLOCATION DRAG COEFFICIENT}

The applied shear stress $\tau$ and the dislocation velocity $v$ are often related by the linear relation

$$
\tau=B v / b
$$

where $b$ is the magnitude of the Burgers vector and $\mathrm{B}$ is a dislocation drag coefficient.

In the simulations the dislocation velocity does not increase proportionally with increasing stress. However, the dislocation drag coefficient in such a relation can, for a given temperature, be estimated by considering the slope of a line radiating from the origin and becoming tangent

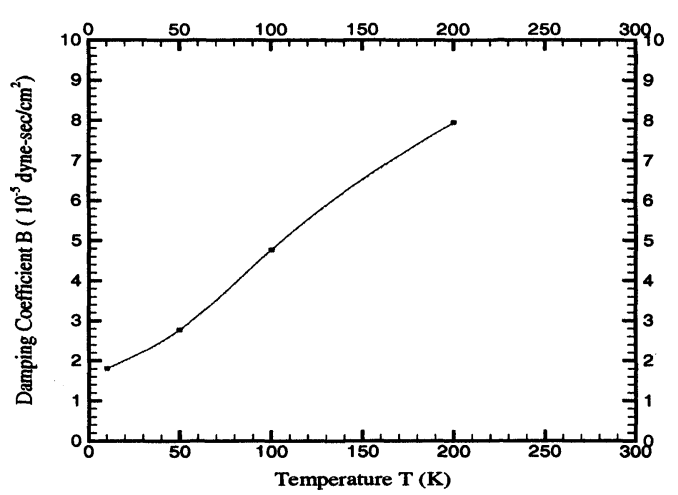

FIGURE 4: Dislocation drag coefficient $B$ as a function of temperature.

to the corresponding curve in Figure 3. Using the tangent line to estimate $B$ minimizes effects from the Peierls stress regime as well as from the regime in which the dislocation velocity is at its subsonic limiting value. The dislocation drag coefficient determined in this way has the temperature dependence shown in Figure 4 . While it is tempting to regard Figure 4 as giving a prediction of the temperature dependence of the drag coefficient B for aluminum, one should remember that the plot of dislocation velocity vs. applied stress in Figure 3 is strongly nonlinear. Thus, Figure 4 should be interpreted as a means of characterizing the dependence of dislocation mobility on temperature over a certain range of stresses, not as support for the validity of the linear drag relation of Eqn. (1).

\section{LATTICE DYNAMICS PREDICTION OF THE LIMITING DISLOCATION VELOCITY}

Groteleuschen $^{10}$ and Gumbsch and $\mathrm{Gao}^{3}$ observed a relationship between dislocation velocity and applied stress that is similar to the one shown in Figure 2. The limiting velocity in the third regime (about $70 \%$ of the slowest shear wave speed) cannot be explained by continuum elasticity. The lattice-dynamics model by Celli and Flytzanis ${ }^{11}$ suggests a means for providing a possible explanation.

Using lattice dynamics with nearest-neighbor interactions, Celli and Flytzanis ${ }^{11}$ derived the 
relation between dislocation velocity and applied strain for a screw dislocation in a twodimensional lattice. The Celli-Flytzanis analysis suggests that the limiting dislocation velocity is a phonon velocity having equal values for its phase velocity and its group velocity, in the direction of the motion of the dislocation. To obtain such a limiting dislocation velocity for aluminum, dispersion relations are obtained using the EAM potentials. These dispersion relations are represented as plots of the frequency $\omega$ as a function of the components $k_{x}, k_{y}$ and $k_{z}$ of the wave number $\mathbf{k}$ of harmonic plane waves.

The equations of motion for atoms in a threedimensional lattice can be expressed as,

$$
M \ddot{\mathbf{u}}(\mathbf{R})=-\sum_{\mathbf{R}^{\prime}} \mathbf{D}\left(\mathbf{R}-\mathbf{R}^{\prime}\right) \mathbf{u}\left(\mathbf{R}^{\prime}\right),
$$

where $M$ is the mass matrix, $\mathbf{u}$ are the displacements and $\mathbf{D}$ is the force-constant matrix formed using the interaction between the atoms of the lattice. Dispersion relations are obtained by considering plane wave solutions for the displacements $\mathbf{u}$ to obtain the characteristic condition

$$
\left|\tilde{\mathbf{D}}-M \omega^{2} \mathbf{I}\right|=0,
$$

where $\tilde{\mathbf{D}}$ is the dynamical matrix. Eq. (3) can be solved numerically to obtain the eigenvalues, $\omega^{2}$ for given k. A numerical program, vibra, developed at Sandia National Laboratories was used for this purpose.

Based on the Celli-Flytzannis analysis, phonons associated with the limiting dislocation velocity, say $v_{1}$, are expected to satisfy $\partial \omega / \partial k_{y}=$ $0, \partial \omega / \partial k_{z}=0, \partial \omega / \partial k_{x}=v_{1}$. For a lattice having fcc symmetry, and for the dislocation orientation of Figure 1, the first two conditions are satisfied for $k_{y}=2 \sqrt{2} /\left(a_{0} \sqrt{3}\right)$ and $k_{z}=$ $2 \pi /\left(a_{0} \sqrt{3}\right)$. For these values of $k_{y}$ and $k_{z}$, Figure 5 shows the dispersion relation for aluminum based on the Ercolessi-Adams potentials. The group velocity and the phase velocity in the $\mathrm{x}$-direction are equal for the wave number at which a radial line from the origin is tangent to one of the dispersion curves. The wave number and velocity at the first such tangent point are

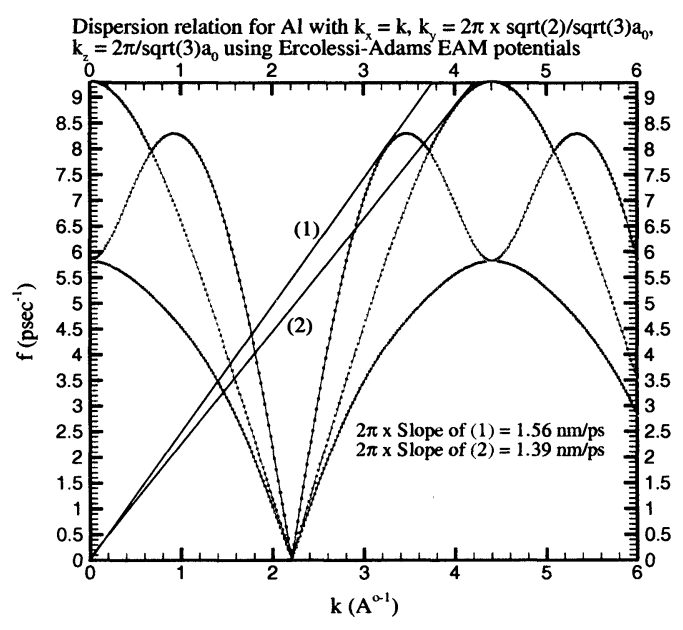

FIGURE 5: Dispersion relation for aluminum, based on Ercolessi-Adams EAM potentials.

$k_{x}=3.28 \AA^{-1}, \quad v_{1}=1.56 \mathrm{~nm} / \mathrm{ps}$. The velocity $v_{1}$ is approximately $25 \%$ less than the velocity at the plateau in Figure 2. Better agreement is obtained as the computational effects of cell size are reduced. For example, doubling and halving the spacing of dislocations in the $\mathrm{x}$-direction leads to an extrapolated value for the plateau velocity of approximately $v_{1}$ as the reciprocal of the dislocation spacing approaches zero.

\section{ACKNOWLEDGEMENTS}

The authors gratefully acknowledge the support of the NSF-MRSEC at Brown University.

\section{REFERENCES}

1. Jiang, C. B., Patu, S., Lei, Q. Z. and Shi, C. X., Philos. Mag. Lett., 78, pp. 1-8 (1998).

2. Gumbsch, P., Riedle, J., Hartmaier, A. and Fischmeister, H. F., Science, 282, pp. 1293-1295 (1998).

3. Gumbsch, P. and Gao, H. Science, 283, pp. 965-968 (1999).

4. Daw, M. S., Foiles, S. M. and Baskes, M. I. Material Science Reports, 9, pp. 251-310 (1993).

5. Grujicic, M., Journal of Materials Science, 32, pp. 1749-1757 (1997).

6. Bhate, N., PhD Thesis, Brown University (2001).

7. Ercolessi, F. and Adams, J. Europhysics Letters, 26 , pp. 583-588 (1993).

8. Hoover, W.G., Computational Statistical Mechanics, Elsevier (1991)

9. Eshelby, J. D., Proc. R. Soc. London A, 62 (1949).

10. Groteleuschen, L. P., PhD Thesis, Brown University (1993).

11. Celli, V. and Flytzanis, N., J. Appl. Phys., 41, pp. 4443-4447 (1970). 